\title{
Low Complexity Multi-Resource Fair Queueing with Bounded Delay
}

\author{
Wei Wang, Ben Liang, Baochun Li \\ Department of Electrical and Computer Engineering \\ University of Toronto
}

\begin{abstract}
Middleboxes are ubiquitous in today's networks. They perform deep packet processing such as content-based filtering and transformation, which requires multiple categories of resources (e.g., CPU, memory bandwidth, and link bandwidth). Depending on the processing requirement of traffic, packet processing for different flows may consume vastly different amounts of resources. Multi-resource fair queueing allows flows to obtain a fair share of these resources, providing service isolation across flows. However, previous solutions for multiresource fair queueing are either expensive to implement at high speeds, or incurring high scheduling delay for flows with uneven weights. In this paper, we present a new fair queueing algorithm, called Group Multi-Resource Round Robin $\left(\mathbf{G M R}^{3}\right)$, that schedules packets in $O(1)$ time, while achieving near-perfect fairness with a low scheduling delay bounded by a small constant. To our knowledge, this work represents the first multi-resource fair queueing algorithm that are provably fair, highly efficient, and with low delays.
\end{abstract}

\section{INTRODUCTION}

Fair queueing algorithms [1], [2], [3], [4], [5] are designed to schedule packets in network switches in a fair and efficient manner, and serve as the foundation of Quality of Service (QoS) research in networking. With fair queueing algorithms, a scheduler determines the order in which packets of various independent flows are forwarded on a shared output link, allocating a fair share of the outgoing bandwidth to each flow.

With the evolution of network appliances, output bandwidth is no longer the only shared resource in today's enterprise networks. Modern network appliances or "middleboxes" do more than just packet forwarding. In addition, they perform filtering (e.g., firewalls), optimization (e.g., HTTP caching and WAN optimization), and transformation (e.g., dynamic request routing) based on traffic contents [6], [7], [8], which require the support of multiple resources such as CPU, memory bandwidth, and link bandwidth [9], [10]. Multi-resource fair queueing algorithms are therefore needed to schedule these resources to meet the QoS requirements of flows.

While fair queueing for bandwidth sharing have been extensively studied [1], [2], [11], [3], [12], [4], multi-resource fair queueing imposes new scheduling challenges as flows are competing for multiple resources and may have vastly different resource requirements. For example, flows that require forwarding a large amount of small packets congest the memory bandwidth of a software router [13], while those that require IP security encryption (IPSec) needs more CPU processing time [14]. Despite their heterogeneous resource requirements, flows are expected to receive predictable service isolation to meet their QoS requirements. This requires a multi-resource packet scheduler with the following three desired properties.
Fairness: The scheduler should provide some measure of service isolation across flows, so that the bad behaviour of rogue traffic will not affect the QoS of regular flows. In particular, each flow should receive service at least at the level when every resource is allocated in proportion to the flow's weight, irrespective of the behaviours of other traffic.

Bounded delay: Interactive Internet applications such as video streaming and online games have stringent end-to-end delay requirements. It is hence important for a scheduler to offer a bounded scheduling delay. Such a delay bound should be a small constant, independent of the number of flows.

Low complexity: As the volume of traffic through middleboxes increases [15], [16], it is important to make scheduling decisions at high speeds. Ideally, a packet scheduler should have a time complexity that is a small constant, independent of the number of flows. In addition, the scheduling algorithm should be amenable to practical implementations.

Despite recent advances in multi-resource fair queueing (e.g., [10]), how a multi-resource packet scheduler is to be designed to satisfy all three desirable properties remains an open and elusive challenge. Existing designs either are expensive to implement at high speeds, or provide no guaranteed delay bound. In particular, DRFQ [10], the first multi-resource fair queueing that implements Dominant Resource Fairness (DRF) [17], associates packets with timestamps, and schedules the one with the earliest timestamp. It suffers from a sorting bottleneck with high scheduling complexity, logarithmic in the number of flows. To avoid the sorting bottleneck of DRFQ, we have designed a simpler scheduler, referred to as $\mathrm{MR}^{3}$, in our previous work [18]. $\mathrm{MR}^{3}$ serves flows in a round-robin fashion, and reduces the scheduling complexity to $O(1)$ time per packet. However, as we shall show in Sec. II, MR ${ }^{3}$ may incur an unbounded delay for weighted flows, and is hence unsuitable for applications with stringent delay requirements.

In this paper, we present a new packet scheduling algorithm, referred to as Group Multi-Resource Round Robin $\left(\mathrm{GMR}^{3}\right)$, that achieves all three desirable properties. GMR ${ }^{3}$ groups flows with similar weights into a small number of groups, each associating with a timestamp. The scheduling decisions are made in a two-level hierarchy. At the higher level, GMR ${ }^{3}$ makes inter-group scheduling decisions by choosing the group with the earliest timestamp, while at the lower level, intragroup scheduling serves flows within a group in a round robin fashion. GMR ${ }^{3}$ is highly efficient, as it requires only a time complexity of $O(1)$ per packet in almost all practical scenarios. In addition, as a highlight of this paper, we show that $\mathrm{GMR}^{3}$ achieves near-perfect fairness across flows, with its scheduling delay bounded by a small constant. These desirable 


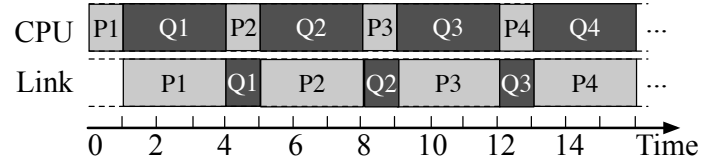

Fig. 1. A schedule that implements DRF, where flow 1 sends packets P1, P2, $\ldots$, while flow 2 sends packets Q1, Q2, ...

properties are not only proven analytically, but also validated experimentally in this paper. To our knowledge, $\mathrm{GMR}^{3}$ is the first multi-resource fair queueing algorithm that offers nearperfect fairness with $O(1)$ time complexity and a constant scheduling delay bound.

The remainder of this paper is organized as follows. We clarify the design objectives and discuss the drawbacks of existing multi-resource queueing schemes in Sec. II. In Sec. III, we present our design of $\mathrm{GMR}^{3}$ algorithm. Theoretical analysis and simulation studies are then given in Sec. IV and V, respectively. Sec. VI concludes the paper.

\section{OBJectives AND Challenges}

In this section, we explain some terminologies and clarify the detailed design objectives of a multi-resource scheduler. We then briefly revisit existing multi-resource fair queueing algorithms and show that they either suffer from high complexity or incur unbounded scheduling delay.

\section{A. Design Objectives}

Dominant Resource Fairness: Fairness is the primary design objective for a packet scheduler. The recently proposed Dominant Resource Fairness (DRF) serves as a promising notion of fairness in a system containing multiple resources [10], [17], [19], [20]. DRF generalizes max-min fairness to the dominant resource in the multi-resource setting [17]. The dominant resource is defined as the one that requires the maximum packet processing time. Specifically, let $m$ be the number of resources under consideration. For packet $p$, let $\tau_{r}(p)$ be the time required to process it on resource $r$. The dominant resource $r^{*}$ of packet $p$ is

$$
r^{*}=\underset{1 \leq r \leq m}{\arg \max } \tau_{r}(p) .
$$

Under DRF, flows receive the same processing time on their dominant resources (assuming flows are of equal weights). For example, consider two flows. Flow 1 requires basic forwarding, where the link bandwidth is the dominant resource, while flow 2 requires security encryption, where CPU is the dominant resource. To achieve DRF, packets should be scheduled in a way such that the link transmission time flow 1 receives is equal to the CPU processing time flow 2 receives. Fig. 1 illustrates such a schedule, where flow 1 sends packets $\mathrm{P} 1, \mathrm{P} 2, \ldots$, while flow 2 sends packets Q1, Q2, ...

It has been shown in [10], [21] that a schedule that achieves DRF allows flows to receive service at least at the same level as when every resource is allocated in proportion to their weights, irrespective of the behaviours of other traffic, which is commonly known as providing service isolation across flows. Moreover, a DRF schedule is work conserving in that no resource that could be used to increase the throughput of a backlogged flow is wasted in idle. DRF hence serves as a promising notion of fairness for multi-resource fair queueing.

To measure how well a packet scheduler implements DRF, the following Relative Fairness Bound (RFB) generalizes the Golestani fairness measure [11] to the multi-resource setting and is used as an important fairness metric [10], [18], [21].

Definition 1: For any packet arrivals and any time interval $\left(t_{1}, t_{2}\right)$, let $T_{i}\left(t_{1}, t_{2}\right)$ be the packet processing time flow $i$ receives on its dominant resource in $\left(t_{1}, t_{2}\right)$, and is referred to as the dominant service. Let $\mathcal{B}\left(t_{1}, t_{2}\right)$ be the set of flows that are backlogged in $\left(t_{1}, t_{2}\right)$. Finally, let $w_{i}$ be the weight of flow $i$. The Relative Fairness Bound is defined as

$$
\mathrm{RFB}=\sup _{t_{1}, t_{2} ; i, j \in \mathcal{B}\left(t_{1}, t_{2}\right)}\left|\frac{T_{i}\left(t_{1}, t_{2}\right)}{w_{i}}-\frac{T_{j}\left(t_{1}, t_{2}\right)}{w_{j}}\right| .
$$

RFB bounds the gap of dominant services received by any two flows in any backlogged period. Intuitively, the smaller the gap, the fairer the scheduler. One of our objectives is to design a scheduler with RFB being a small constant.

Scheduling Delay: In addition to fairness, scheduling delay is another important concern for a packet scheduler. The scheduling delay is defined as the time that elapses between the instance a packet reaches the head of its queue, and the instance the packet finishes being processed on all resources. The delay is introduced by the scheduling algorithm and is also referred to as single packet delay in the fair queueing literature [22], [23], [24], [25]. Intuitively, flows with larger weights are expected to experience smaller delay. In the ideal case, the delay $d$ experienced by a flow $i$ should be within a small constant amount that is inversely proportional to the deserved processing rate (weight) of the flow, i.e.,

$$
d \leq C / w_{i}
$$

where $C$ is a constant.

Scheduling Complexity: To handle a large volume of traffic at high speeds, the scheduler must operate with low scheduling complexity, defined as the time required to make a packet scheduling decision. Ideally, this complexity should be a small constant, independent of the number of flows.

In summary, a good packet scheduler should offer nearperfect fairness and a constant delay bound that is inversely proportional to the flow's weight, while operating in $O(1)$ time complexity as well. Unfortunately, none of the existing design provides all these properties, as we shall see in the next subsection.

\section{B. Previous Work and Challenges}

There are two alternative approaches that existing multiresource fair queueing algorithms use in their design. Timestamp-based algorithms (e.g., [10], [21]) associate timestamps with each packet upon its arrival. Whenever there is a scheduling opportunity, the packet with the earliest timestamp is scheduled. Since these schedulers need to sort packet timestamps, they suffer from high scheduling complexity, requiring $O(\log n)$ time per packet, where $n$ is the number of flows. This sorting bottleneck significantly limits the scalability of 


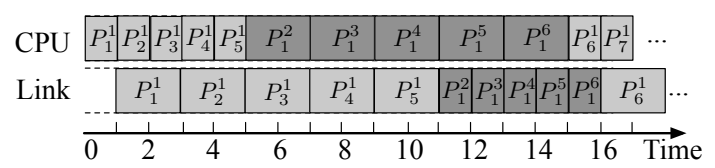

Fig. 2. $\mathrm{MR}^{3}$ schedule fails to offer weight-proportional delay when flows are assigned uneven weights. $P_{k}^{i}$ denotes the $k$ th packet of flow $i$.

these algorithms, and necessitates a simpler scheduler. As an alternative, Multi-Resource Round Robin ( $\mathrm{MR}^{3}$ ) [18] serves flows in rounds. Each flow maintains a credit account, and in each round, a certain amount of credit that is proportional to the flow's weight is given. The amount of available credit represents the dominant service the flow is allowed to consume in one round. A flow can overdraw its credit, and the excessive consumption will be deducted from the credit given in the next round. $\mathrm{MR}^{3}$ eliminates the sorting bottleneck and requires only $O(1)$ complexity per packet [18].

However, $\mathrm{MR}^{3}$ fails to offer a weight-proportional delay bound. To see this, consider an example where 6 flows are competing for both middlebox CPU and the link bandwidth. Each packet of flow 1 requires 1 time unit for CPU processing and 2 for link transmission. Each packet of other flows requires 2 time unit for CPU processing and 1 for link transmission. Flow 1 weighs $1 / 2$, while flow 2 to 6 each weighs $1 / 10$. The amount of credits flow 1 receives in one round is hence 5 times those given to the other flows. Fig. 2 illustrates an $\mathrm{MR}^{3}$ schedule, where $P_{k}^{i}$ denotes the $k$ th packet of flow $i$. We see that the schedule offers weight-proportional services but not weight-proportional delay. The maximum packet scheduling delay flow 1 experiences is 13 time units (e.g., packet $P_{6}^{1}$ ), more than half of that experienced by other flows (e.g., packet $P_{2}^{2}$ has been delayed by 20 time units).

Formally, the following analyses show that $\mathrm{MR}^{3}$ may incur unbounded scheduling delay when flows are assigned uneven weights. Let $W$ be the maximum ratio between weights of two flows, i.e.,

$$
W=\max _{i, j} w_{i} / w_{j} .
$$

The following theorem bounds the single packet delay of $\mathrm{MR}^{3}$. The proof is deferred to our technical report [26].

Theorem 1: Under $\mathrm{MR}^{3}$, for any flow $i$, the scheduling delay of its packet $P$ is bounded by

$$
D(P)<4(m+W)^{2} L / w_{i},
$$

where $L$ is the maximum packet processing time across flows, and $m$ is the number of resources under consideration.

By Theorem 1, we see that the delay bound of $\mathrm{MR}^{3}$ critically depends on the weight distributions and may become arbitrarily large when $W \gg 1$. To summarize, it remains open to design a multi-resource packet scheduler that offers nearperfect fairness with low complexity and small delay bound.

Similar complexity and delay issues have also been a major challenge in the long evolution of single-resource fair queueing algorithms for bandwidth sharing, where both timestampbased schemes and round robin are the two basic approaches in the design. The former provides good delay bounds yet requires high complexity to sort packet timestamps (e.g., [1], [2], [11], [12], [5]). The later approach, on the other hand, has

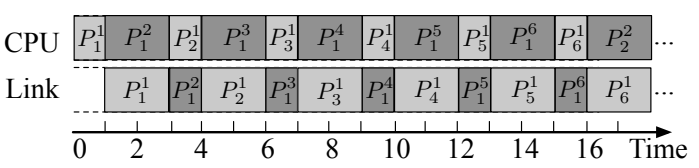

Fig. 3. An improved schedule over $\mathrm{MR}^{3}$ in Fig. 2, where the scheduling delay is significantly reduced. $P_{k}^{i}$ denotes the $k$ th packet of flow $i$.

$O(1)$ complexity, yet incurs high scheduling delays (e.g., [4], [27], [23]). To achieve the best of both worlds, one approach is to combine fairness and delay properties of timestamp-based algorithms with low time complexity of round-robin schemes. This is typically done by grouping flows into a small number of classes. The scheduler then uses the timestamp-based algorithm to determine which class to serve. Within a class, the scheduling resembles a round-robin scheme. While this strategy turns out to be an effective approach for bandwidth sharing [22], [28], [29], [24], [25], generalizing it to schedule multiple resources imposes non-trivial technical challenges. Given that flows may have different dominant resources, the scheduler has to maintain a consistent service level across all these resources. We answer this challenge in the next section.

\section{Group Multi-Resource Round Robin}

In this section, we present our design of Group MultiResource Round Robin $\left(\mathrm{GMR}^{3}\right)$ that provides all the desirable scheduling properties defined in Sec. II.

\section{A. Basic Intuition}

While round robin may incur high delay in a general scenario, Theorem 1 indicates that it provides a good delay bound when flows are of similar weights (within a small constant factor of $W$ ). In other words, if we group flows with similar weights to a flow group, then within the group, round robin serves as an excellent scheduler. The challenge is to schedule inter-group flows with different weights. Recall that in $\mathrm{MR}^{3}$, flows are always served in a "burst" mode [18]. For example, in Fig. 2, flow 1 schedules 5 packets in a row in round 1 , and has to wait for an entire round to schedule its next packet $P_{6}^{1}$ in round 2 , resulting in a long scheduling delay of that packet.

Instead of serving flows in a "burst" mode, a better strategy is to spread their scheduling opportunities over time, in proportion to their respective weights. Fig. 3 illustrates an improved schedule over $\mathrm{MR}^{3}$ in Fig. 2, where the scheduling opportunities of flow 1 are interleaved between those of other flows. Compared to $\mathrm{MR}^{3}$ schedule in Fig. 2, the maximum scheduling delay of flow 1 is significantly reduced from 13 to 5 , and the delay of other flows is also reduced from 20 to 16.

Our design follows exactly this intuition. The algorithm groups flows with similar weights to a flow group, and makes scheduling decisions in a two-level hierarchy. At a higher level, the algorithm makes inter-group scheduling decisions to determine a flow group, with the objective of distributing the scheduling opportunities over time, in proportion to the approximate weights of flows. Within a group, the intra-group scheduler serves flows in a round-robin fashion. We shall show in Sec. IV that this simple combination leads to remarkable 
performance guarantees. For now, we focus on the detailed design in the following subsections.

\section{B. Flow Grouping}

Suppose there are $n$ backlogged flows sharing $m$ middlebox resources. Without loss of generality, let the flow weight $w_{i}$ be normalized such that

$$
\sum_{i=1}^{n} w_{i}=1 .
$$

The scheduler groups flows with similar weights to a flow group. Specifically, flow group $G_{k}$ is defined as

$$
G_{k}=\left\{i: 2^{-k} \leq w_{i}<2^{-k+1}\right\}, \quad k=1,2, \ldots
$$

Thus, the weights of any two flows belonging to the same flow group are within a factor of 2 of each other.

Such a grouping strategy leads to a small number of flow groups $n_{g}$, bounded by $n_{g} \leq \log _{2} W$. As pointed out in [22], [24], [25], for a practical flow weight distribution, the number of flow groups $n_{g} \leq 40$ and can hence be safely assumed as a small constant. This significantly reduces the complexity of the inter-group scheduling.

\section{Inter-Group Scheduling}

The inter-group scheduler determines a flow group to potentially schedule a flow. Each group is associated with a timestamp, and the one with the earliest timestamp is selected. With appropriate timestamps, the scheduling opportunities of a flow group would be weight-proportionally distributed over time. Given that the number of groups $n_{g}$ is a small constant, the complexity of sorting the group timestamps is also a small constant $O\left(\log n_{g}\right)$. Among various timestampbased algorithms, we find that [22] is particularly attractive for multi-resource extension, due to its simple timestamp computation. Extending other algorithms (e.g., [24], [25]) to multiple resources would require referring to the idealized fluid DRGPS model [21], incurring high complexity.

The scheduler maintains an accounting mechanism consisting of a sequence of virtual slots, indexed by $0,1,2$, .... Each slot is exclusively assigned to one flow, and is the scheduling opportunity of this flow. Each flow group $G_{k}$ is associated with a set of scheduling rounds each spanning $2^{k}$ contiguous slots. The first scheduling round of flow group $G_{k}$, denoted $R_{1}^{k}$, starts at slot 0 and ends at slot $2^{k}-1$, while the second scheduling round, denoted $R_{2}^{k}$, starts at slot $2^{k}$ and ends at slot $2^{k+1}-1$, and so on. Fig. 4 gives an example. Note that the scheduling rounds of different flow groups overlap by design. The scheduler assigns each backlogged flow $i \in G_{k}$ exactly one slot per scheduling round of flow group $G_{k}$. This allows flow $i$ to receive one scheduling opportunity every $2^{k}$ slots, roughly matching the flow's weight (i.e., $2^{-k} \leq w_{i}<2^{-k+1}$ ). The scheduling opportunities of flows are hence weight-proportionally distributed over time.

Following [22], a flow group is called active if it contains at least one backlogged flow. A backlogged flow $i \in G_{k}$ is called pending if it has not yet been assigned a slot in the current scheduling round of $G_{k}$. A flow group is called pending if it contains at least one pending flow.

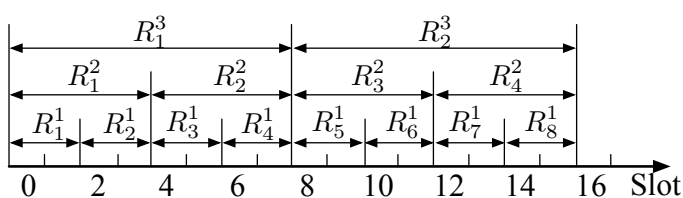

Fig. 4. An illustration of the scheduling rounds of flow groups, where $R_{l}^{k}$ denotes the scheduling round $l$ of flow group $G_{k}$.

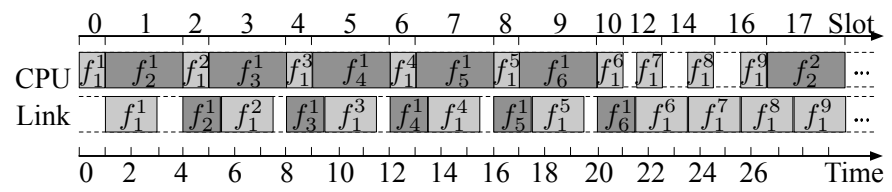

Fig. 5. An illustration of $\mathrm{GMR}^{3}$ scheduler assigning slots to flows in the example of Fig. 2, where $f_{i}^{l}$ denotes the packet processing for flow $i \in G_{k}$ in the scheduling round $l$ of its flow group $G_{k}$.

For every virtual slot $t$, the inter-group scheduler chooses among all pending flow groups the one with the earliest timestamp, defined as the ending slot of the current scheduling round of that flow group. Ties are broken arbitrarily. From the selected flow group, the intra-group scheduler then chooses a pending flow and assigns it the current slot $t$ (with details to be described in Sec. III-D). A flow temporarily ceases to be pending once it has been assigned a slot in the current scheduling round of its flow group, and will become pending again at the beginning of the next scheduling round, if remaining backlogged. If no group is pending in slot $t$, the slot is skipped. Algorithm 1 summarizes this inter-group scheduling process.

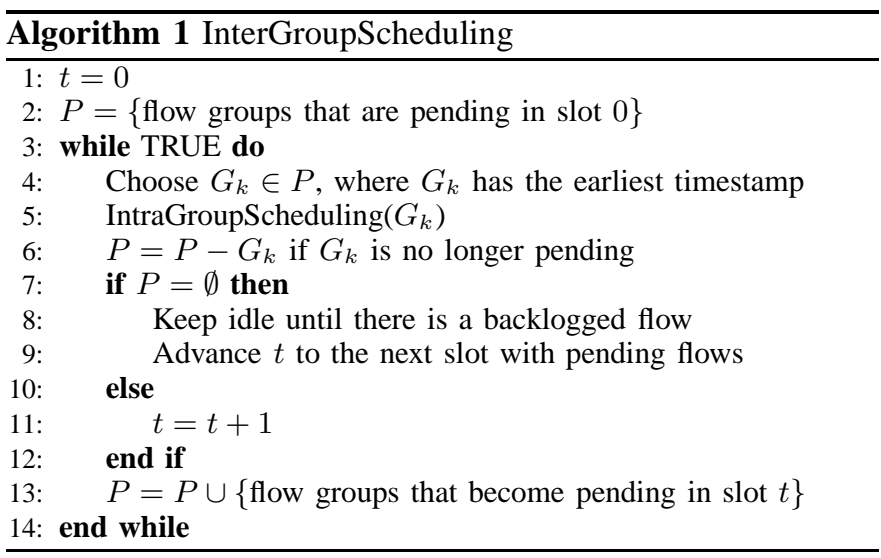

Fig. 5 illustrates an example of the inter-group scheduler assigning slots to flows in the example of Fig. 2, where $f_{i}^{l}$ denotes the packet processing for flow $i$ in the scheduling round $l$ of its flow group. Note that the slot axis is only for the accounting mechanism, while the time axis shows the real time elapse. Flow 1 belongs to $G_{1}$ as its weight is $1 / 2$, while flows 2 to 6 are grouped to $G_{4}$ as each of their weights is $1 / 10$. At slot 0 , both $G_{1}$ and $G_{4}$ are pending, with the end of the current scheduling round at slot 1 and slot 15 , respectively. The inter-group scheduler hence picks $G_{1}$, from which the intragroup scheduler selects flow 1 as it is the only backlogged flow in $G_{1}$. Flow 1 then schedules its packets for processing and ceases to be pending in the current scheduling round. As 
a result, in slot 1 , only $G_{4}$ is pending and flow 2 is assigned the slot. Flow 1 becomes pending again in slot 2 as a new scheduling round of its flow group $G_{1}$ starts, and is selected for the similar reason as in slot 0 . Flow groups $G_{1}$ and $G_{4}$ are hence selected alternately in the following slots until all flows of $G_{4}$ are assigned slots and cease to be pending. Note that slots 11, 13, and 15 are not shown in Fig. 5 as no flow is pending in these slots, and are hence skipped by the scheduler.

Unlike the single-resource scheduling [22], in the multiresource environment, a flow may not receive dominant services in its assigned slots. For example, in Fig. 5, flow 1 is assigned slot 0 , but receives dominant services (i.e., link transmission) later in slot 1 . Flow 2, on the other hand, always receives dominant services (i.e., CPU processing) in its assigned slots. Without appropriate control, the potential service asynchronicity may lead to a significant work progress gap between two resources, resulting in the poor fairness and long scheduling delay. We show in the next subsection that this problem is effectively solved by the intra-group scheduler.

\section{Intra-Group Scheduling}

Once the flow group is determined, the intra-group scheduler chooses a pending flow from that group in a round-robin manner. Compared to round robin for bandwidth sharing (e.g., [4], [23], [27], [29], [22], [24], [25]), the intra-group scheduler operates with two important differences. First, for the purpose of DRF, the scheduler maintains a credit system to keep track of the dominant services a flow receives, not the amount of bits a flow transmits. Second, the scheduler employs a progress control mechanism to reinforce a relatively consistent work progress across resources, so as to eliminate the adverse effects caused by the aforementioned service asynchronicity.

Credit System: Every time a flow $i$ is assigned a slot, it receives a credit $c_{i}$ (whose size is given in (4) below), which is the time given to the flow for packet processing on its dominant resource in the current scheduling round. As long as there are available credits, flow $i$ is allowed to schedule a packet for processing, and the corresponding packet processing time on the dominant resource is deducted from its total credit. A flow $i$ can overdraw the processing time by scheduling at most one more packet than those allowed by the available credits. The excessive consumption of dominant services is tracked by the excess counter $e_{i}$, and will be deducted from the credit given in the next scheduling round as a penalty of overconsumption.

While $\mathrm{MR}^{3}$ adopts a similar credit system in its design [18], the intra-group scheduler of $\mathrm{GMR}^{3}$ operates with an important difference. Every time a flow $i$ is assigned a slot, instead of receiving an elastic amount of credits in different rounds, it is given a fixed-size credit that is proportional to its weight $w_{i}$. Specifically, for flow $i \in G_{k}$, the given credit $c_{i}$ is

$$
c_{i}=2^{k} L w_{i},
$$

where $L$ is the maximum packet processing time. The motivation for defining credit in this manner is two-fold.

To begin with, even if two flows $i, j$ belong to the same group $G_{k}$, flow $i$ 's weight $w_{i}$ may be up to twice as large as $w_{j}$. Despite their weight difference, both flows are assigned exactly 1 slot per scheduling round of $G_{k}$. Therefore, to ensure weight-proportional dominant services, the given credits as shown in (4) are proportional to their respective weights.

Moreover, for each flow $i \in G_{k}$, since $2^{-k} \leq w_{i}<2^{-k+1}$, the scaling factor $2^{k} L$ in (4) ensures that

$$
L \leq c_{i}<2 L
$$

Because the given credits are larger than the maximum packet processing time, they can always compensate for the overconsumption of dominant services flow $i$ incurs in the previous scheduling round. As a result, flow $i$ will always have available credits when assigned a slot, and can schedule at least one packet. In addition, by (5), the given credits are roughly the same across all flow groups. This is significant as flow $i \in G_{k}$ is already assigned slots in proportion to its approximate weight $2^{-k}$, so that in each slot, the scheduler should allocate all flows approximately the same dominant services.

Progress Control Mechanism: In addition to the credit system, the scheduler also employs a progress control mechanism to reinforce a relatively consistent processing rate across resources. Specifically, whenever a flow $i \in G_{k}$ is assigned a slot $t$ in the scheduling round $l$ of $G_{k}$, the scheduler checks the work progress on the last resource (usually the link bandwidth). If flow $i$ has already received services on the last resource in the previous scheduling round $l-1$, or flow $i$ is a new arrival, then its packet is scheduled immediately. Otherwise, the scheduler defers packet scheduling until flow $i$ starts to receive service on the last resource in the previous scheduling round $l-1$ of $G_{k}$. For example, as shown in Fig. 5, in slot 12 , the packet processing for flow 1 (i.e., $f_{1}^{7}$ ) is withheld in round 7 until the packet processed in round 6 (i.e., $f_{1}^{6}$ ) starts transmission. Similar deferral has also been shown in slots 14 and 16. Intuitively, this progress control mechanism ensures that the work progress on one resource is not ahead of that on the other by more than 1 round, hence achieving an approximately consistent processing rate across resources, in spite of the potential service asynchronicity. This progress control mechanism is essential to deriving the constant delay bound of GMR3, as shown in our analysis in Sec. IV.

To summarize, Algorithm 2 gives detailed design of the intra-group scheduling. Every flow group $G_{k}$ maitains an ActiveFlowList $[k]$ for its backlogged flows. It also uses RoundRobinCounter $[k]$ and Round $[k]$ to keep track of the current scheduling round. Every time flow group $G_{k}$ is selected, the intra-group scheduler chooses flow $i \in G_{k}$ at the head of ActiveFlowList $[k]$. Flow $i$ is given a credit to compensate for its overdraft in the previous round, and schedule packets until no credit remains or no packet is backlogged (line 6 to 15). After that, the flow ceases to be pending and is appended to the tail of the active list if it remains backlogged. Flow group $G_{k}$ ceases to be pending when all its backlogged flows are serviced in the current scheduling round. If no flow is backlogged, flow group $G_{k}$ becomes inactive.

\section{E. Handling New Packet Arrivals}

In addition to determining the packet scheduling order, $\mathrm{GMR}^{3}$ scheduler also needs to handle new packet arrivals. 


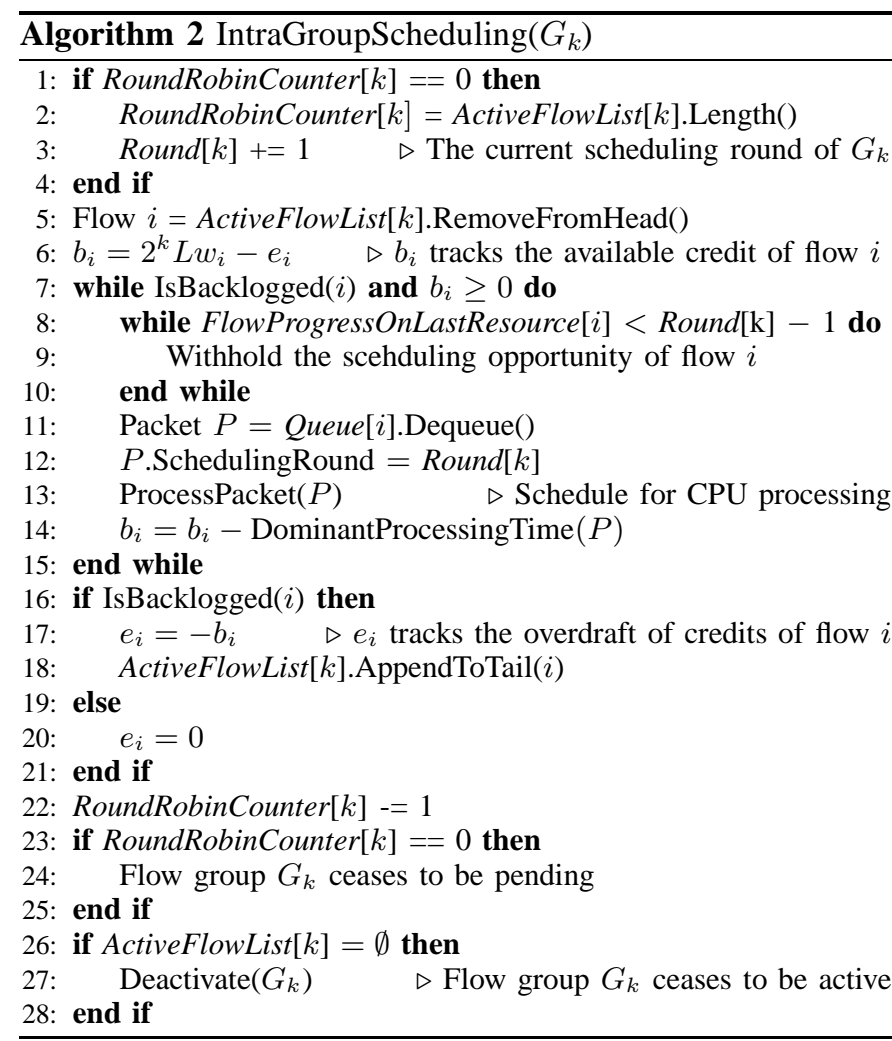

Algorithm 3 gives the detailed procedure. In addition to enqueueing the newly arrived packet $p$ to the queue of flow $i \in G_{k}$ to which the packet belongs, the scheduler also appends flow $i$ to the active list of its flow group $G_{k}$ if flow $i$ is previously inactive. Flow group $G_{k}$ is also activated if it is inactive before.

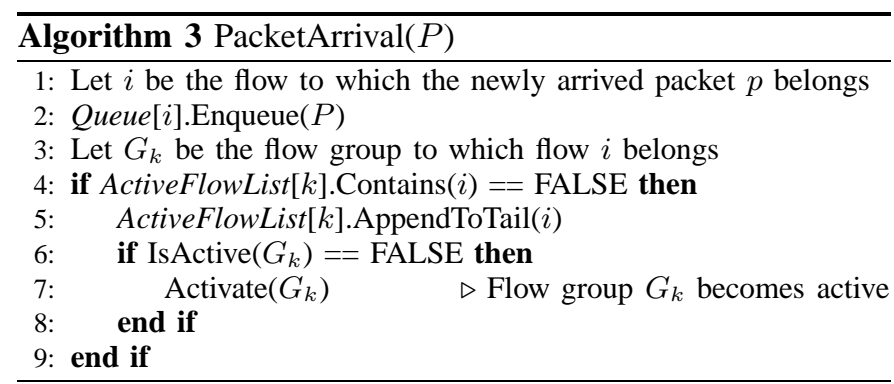

\section{F. Implementation and Complexity}

So far, we have described the design of $\mathrm{GMR}^{3}$. In this subsection, we show that appropriate implementations to both the inter-group and intra-group scheduling allows $\mathrm{GMR}^{3}$ to make packet scheduling decisions in $O(1)$ complexity.

Inter-Group Scheduling: There are three important operations in Algorithm 1, i.e., choosing a flow group (line 4), advancing to the earliest slot with pending groups (line 9), and updating the pending set $P$ (line 13). Given a small number of flow groups $n_{g}$, all these operations can be done in $O(1)$ time using the simple methods described in [22], which we briefly mention in the following.

The scheduler uses two bitmaps $a=a_{n_{g}} \ldots a_{2} a_{1}$ and $p=$ $p_{n_{g}} \ldots p_{2} p_{1}$ to track the active and pending flow groups. Bit $a_{k}$ is set to 1 if flow group $G_{k}$ is active, and 0 otherwise. Similarly, bit $p_{k}$ is 1 if group $G_{k}$ is pending, and 0 otherwise.

Choosing a flow group: It is easy to check that, in all slot $t$, the scheduling round of flow group $G_{k}$ ends earlier than those of all flow groups $G_{k^{\prime}}$, where $k^{\prime}>k$ (see Fig. 4). Flow group $G_{k}$ hence has a higher priority to be chosen than $G_{k^{\prime}}$. As a result, the chosen group $G_{k}$ can be identified by locating the rightmost bit $p_{k}$ of bitmap $p$ that is set to 1 . Such an operation can be done in $O(1)$ by a standard priority encoder [22].

Advancing to the earliest slot with pending groups: Because the start of the scheduling round for group $G_{k}$ is also the start of a scheduling round of all groups $G_{k^{\prime}}$, where $k^{\prime}>k$ (see Fig. 4), the scheduler should advance to the start of the next scheduling round of the lowest-numbered flow group that is active. This can be identified by locating the rightmost bit $a_{k}$ that is set to 1 , and the new slot is the smallest multiple of $2^{k}$ greater than the current slot $t$. With the surport of priority encoder, all these operations are done in $O(1)$ time.

Updating the pending set: At slot $t$, an active flow group $G_{k}$ becomes pending if $2^{k}$ divides $t$. To identify all these groups, it is sufficient to locate the least significant bit of $t$ that is set to 1 . Let it be the $k$ th least significant bit of $t$. Then all active flow groups $G_{k^{\prime}}$ where $k^{\prime} \leq k$ become pending at $t$, and can be found via some simple bit operations in $O(1)$ [22].

Intra-Group Scheduling: In Algorithm 2, an essential operation is to track the work progress on the last resource of the selected flow $i$ (line 8 to 10) to determine if the scheduling opportunity of flow $i$ should be withheld. For the purpose of efficient implementation, a packet $P$ of flow $i$, upon scheduling, is associated a tag recording the current scheduling round of flow group $G_{k}$ to which flow $i$ belongs (line 12 of Algorithm 2). Whenever packet $P$ starts processing on the last resource $m$, the progress of flow $i$ on that resource is updated as the scheduling round tagged to packet $P$, which will be used later to determine the timing of withholding packet processing of flow $i$ (line 8). All these operations can be done in $O(1)$.

Another operations that may introduce additional complexity is to obtain the packet processing time on the dominant resource (line 14). Note that such information is required only after the packet has been processed by CPU. At that time the scheduler knows exactly how the packet should be processed next and what resources are required. The packet processing time on each of the following resource can hence be accurately inferred via some simple packet profiling techniques in $O(1)$. For example, a simple linear model based on the packet size is proved to be sufficiently accurate for estimation [10].

To conclude, with appropriate implementations mentioned above, both inter-group and intra-group scheduling decisions can be made in $O(1)$ time per packet, making $\mathrm{GMR}^{3}$ a highly efficient multi-resource scheduler for middleboxes.

\section{Performance Analysis}

In this section, we analyze the properties of $\mathrm{GMR}^{3}$ and show that it achieves near-perfect fairness with scheduling delays bounded by a small constant. 


\section{A. Fairness}

For the purpose of fairness analysis, we derive the RFB of $\mathrm{GMR}^{3}$ defined in Sec. II. We start by bounding the dominant services a flow receives in any backlogged period $\left(t_{1}, t_{2}\right)$ as follows. The complete proof is deferred to [26].

Lemma 1: Let $T_{i}\left(t_{1}, t_{2}\right)$ be the dominant service a backlogged flow $i$ receives in a time interval $\left(t_{1}, t_{2}\right)$. We have

$$
x L w_{i}-9 L \leq T_{i}\left(t_{1}, t_{2}\right) \leq x L w_{i}+9 L,
$$

where $x$ is the minimum number of contiguous slots that completely contain $\left(t_{1}, t_{2}\right)$.

Proof sketch: Let $x_{i}$ be the number of slots assigned to flow $i \in G_{k}$ in $\left(t_{1}, t_{2}\right)$. By Algorithm 2, the progress gap between any two resources is upper bounded by 1 scheduling round. It is hence easy to verify that flow $i$ receives services on its dominant resource at least in $x_{i}-2$ scheduling rounds, and at most in $x_{i}+2$ scheduling rounds. The dominant services flow $i$ receives are hence at least $\left(x_{i}-2\right) c_{i}-L$ and are at most $\left(x_{i}+2\right) c_{i}+L$, where $c_{i}=2^{k} L w_{i}$ is the credit given to flow i, i.e.,

$$
\left(x_{i}-2\right) c_{i}-L \leq T_{i}\left(t_{1}, t_{2}\right) \leq\left(x_{i}+2\right) c_{i}+L .
$$

Also, the number of scheduling rounds of flow group $G_{k}$ contained in $\left(t_{1}, t_{2}\right)$ is at least $x_{i}-2$, and is at most $x_{i}+2$. Because each scheduling round of $G_{k}$ spans exactly $2^{k}$ slots, we have $2^{k}\left(x_{i}-2\right) \leq x \leq 2^{k}\left(x_{i}+2\right)$, which is equivalent to

$$
2^{-k} x-2 \leq x_{i} \leq 2^{-k} x+2 \text {. }
$$

Substituting (8) to (7), we derive

$$
\begin{aligned}
T_{i}\left(t_{1}, t_{2}\right) & \leq\left(x_{i}+2\right) c_{i}+L \\
& \leq\left(2^{-k} x+4\right) c_{i}+L \\
& =2^{-k} x 2^{k} L w_{i}+4 c_{i}+L \\
& \leq x L w_{i}+9 L
\end{aligned}
$$

Similarly, we also derive

$$
T_{i}\left(t_{1}, t_{2}\right) \geq\left(x_{i}-2\right) c_{i}-L \geq x L w_{i}-9 L .
$$

Combining (9) and (10) leads to the statement.

We are now ready to derive the RFB of $\mathrm{GMR}^{3}$ as follows.

Theorem 2: For any time interval $\left(t_{1}, t_{2}\right)$ and any two flows $i, j$ that are backlogged, we have

$$
\left|\frac{T_{i}\left(t_{1}, t_{2}\right)}{w_{i}}-\frac{T_{j}\left(t_{1}, t_{2}\right)}{w_{j}}\right| \leq 9 L\left(\frac{1}{w_{i}}+\frac{1}{w_{j}}\right) .
$$

Proof: For any flow $i$, applying Lemma 1 and dividing both sides of (6) by $w_{i}$, we have

$$
x L-9 L / w_{i} \leq T_{i}\left(t_{1}, t_{2}\right) / w_{i} \leq x L+9 L / w_{i} .
$$

Similarly inequalities also hold for flow $j$, i.e.,

$$
x L-9 L / w_{j} \leq T_{j}\left(t_{1}, t_{2}\right) / w_{j} \leq x L+9 L / w_{j} .
$$

Taking the difference between (11) and (12) gives the statement.

Theorem 2 indicates that $\mathrm{GMR}^{3}$ bounds the difference between the normalized dominant services received by two flows in any backlogged period by a small constant. GMR ${ }^{3}$

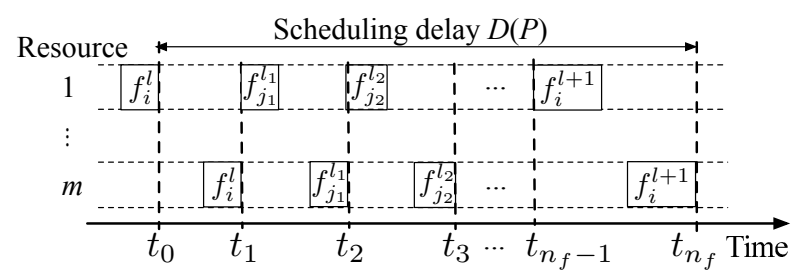

Fig. 6. The illustration of a scenario where the scheduling delay $D(P)$ reaches the maximum. Here, $f_{i}^{l}$ denotes the processing of flow $i$ in scheduling round $l$ of its flow group.

hence provides near-perfect fairness across flows, irrespective of their traffic patterns. This is significant as the fairness guarantees provided by existing multi-resource fair queueing schemes, e.g., [10], [18], all assume flows do not change their dominant resources throughout the backlogged periods (a.k.a., the resource monotonicity assumption [10]).

\section{B. Scheduling Delay}

In addition to the fairness guarantees, we show that $\mathrm{GMR}^{3}$ ensures that the scheduling delay is bounded by a small constant that is inversely proportional to the flow's weight. To see this, the following two lemmas are needed in the analysis. Their proofs are deferred to [26].

Lemma 2: Let $d_{i}^{l}$ be the dominant services flow $i \in G_{k}$ receives in scheduling round $l$ of $G_{k}$. We have

$$
0 \leq d_{i}^{l} \leq 3 L
$$

Lemma 3: For flow $i \in G_{k}$ and scheduling round $l$ of $G_{k}$, let $t_{0}$ be the time when flow $i$ finishes being processed on resource 1 in round $l$ of $G_{k}$, and $t_{1}$ the time when flow $i$ finishes being processed on the last resource $m$ in round $l$. We have

$$
t_{1}-t_{0}<12 m L / w_{i} .
$$

We now bound the scheduling delay of $\mathrm{GMR}^{3}$ as follows.

Theorem 3: For all flow $i$, the scheduling delay of its packet $P$ is bounded by

$$
D(P)<24 m L / w_{i},
$$

where $m$ is the number of resources.

Proof: For any flow $i \in G_{k}$, the scheduling delay of its packet $P$ reaches its maximum when $P$ reaches the head of the queue in scheduling round $l$ of $G_{k}$, but is processed in the next round $l+1$. Since there are at most $2^{k+1}$ slots in between and each slot is assigned to one flow, the number of flows served during this time, $n_{f}$, is upper bounded by $2^{k+1}$. Let these flows be $j_{1}, \ldots, j_{n_{f}}$ operating in their respective current scheduling rounds $l_{1}, \ldots, l_{n_{f}}$. In particular, $j_{n_{f}}=i$ and $l_{n_{f}}=l+1$. By Algorithm 2, flow $j_{1}$ starts service on resource 1 no later than the time its previous flow $i$ finishes being processed on the last resource $m$ in round $l$. Similarly, flow $j_{2}$ starts its service on resource 1 no later than the time its previous flow $j_{1}$ finishes being processed on the last resource $m$ in round $l_{1}$, and so on. Fig. 6 illustrates this scenario, where $t_{z}$ is the latest time flow $j_{z}$ receives service on resource 1 in round $l_{z}$ of its flow group, $z=1,2, \ldots$ We then have

$$
t_{z+1}-t_{z} \leq m d_{j_{z}}^{l_{z}} \leq 3 L m, \quad z=1,2, \ldots,
$$


TABLE I

SUMMARY OF PERFORMANCE OF GMR ${ }^{3}$ AND EXISTING SCHEMES, WHERE $n$ IS THE NUMBER OF FLOWS, AND $m$ IS THE NUMBER OF RESOURCES.

\begin{tabular}{|c||c|c|c|}
\hline Scheme & Complexity & Fairness $^{1}$ & Scheduling Delay \\
\hline DRFQ [10] & $O(\log n)$ & $L\left(1 / w_{i}+1 / w_{j}\right)$ & Unknown \\
\hline MR $^{3}[\mathbf{1 8}]$ & $O(1)$ & $2 L\left(1 / w_{i}+1 / w_{j}\right)$ & $4(m+W)^{2} L / w_{i}$ \\
\hline GMR $^{3}$ & $O(1)$ & $9 L\left(1 / w_{i}+1 / w_{j}\right)$ & $24 m L / w_{i}$ \\
\hline
\end{tabular}

where the second inequality is derived from Lemma 2. In other words, the time span of processing flow $j_{z}$ on all resources in round $l_{z}$ reaches its maximum when the processing time is maximized on every resource.

Now let $t_{0}$ be the time when packet $P$ reaches the head of the queue in scheduling round $l$ of its flow group, which is also the time when flow $i$ finishes being processed on resource 1 in round $l$ (see Fig. 6). By Lemma 3, we have

$$
t_{1}-t_{0} \leq 12 m L / w_{i} \text {. }
$$

With (14) and (15), we bound the delay $D(P)$ as follows:

$$
\begin{aligned}
D(P) & \leq \sum_{z=1}^{n_{f}}\left(t_{z}-t_{z-1}\right) \\
& <12 m L / w_{i}+3 L m n_{f} \\
& \leq 12 m L / w_{i}+3 L m 2^{k+1} \\
& \leq 24 m L / w_{i}
\end{aligned}
$$

where the last inequality holds because $2^{-k} \leq w_{i}<2^{-k+1}$, which implies $2^{k+1} \leq 4 / w_{i}$.

Theorem 3 gives a strictly weight-proportional scheduling delay bound that is independent of the number of flows. This implies that a flow is guaranteed to be scheduled within a small constant amount of time that is inversely proportional to the processing rate (weight) the flow deserves, irrespective of the behaviours of other flows. To our knowledge, this is the first multi-resource packet scheduler that offers this property.

To conclude, Table I compares the performance of $\mathrm{GMR}^{3}$ with DRFQ [10] and $\mathrm{MR}^{3}$ [18]. We see that $\mathrm{GMR}^{3}$ is the only scheduler that provides provably good performance guarantees on fairness, delay, and complexity.

\section{Simulation Results}

For complementary study to our theoretical analysis, we experimentally evaluate the fairness and delay performance of $\mathrm{GMR}^{3}$ via simulations.

General Setup: All simulation results are based on our event-driven packet simulator written with 3,000 lines of $\mathrm{C}++$ code. Packets follow Poisson arrivals and are processed serially on resources, with CPU processing first, followed by link transmission. In addition to $\mathrm{GMR}^{3}$, we also implement DRFQ [10] and $\mathrm{MR}^{3}$ [18] for the purpose of comparison. The simulator simulates packet processing in 3 typical middlebox modules, i.e., basic forwarding (Basic), statistical monitoring (Stat. Mon.), and IP security encryption (IPSec). The first two modules are bandwidth intensive, with monitoring consuming slightly more CPU resources, while IPSec is CPU intensive. According to the measurement results reported in [10], the CPU processing time required by each middlebox module

\footnotetext{
${ }^{1}$ The fairness analysis of DRFQ and $\mathrm{MR}^{3}$ requires that flows do not change their dominant resources throughout the backlogged periods [10], [18].
}

TABLE II

PARAMETERS OF LINEAR MODEL FOR CPU PROCESSING TIME IN 3 MIDDLEBOX MODULES BASED ON MEASUREMENT RESULTS IN [10]

\begin{tabular}{|c|c|}
\hline Module & CPU processing time $(\mu s)$ \\
\hline Basic Forwarding & $0.00286 \times$ PacketSizeInBytes +6.2 \\
\hline Statistical Monitoring & $0.0008 \times$ PacketSizeInBytes +12.1 \\
\hline IPSec Encryption & $0.015 \times$ PacketSizeInBytes +84.5 \\
\hline
\end{tabular}

follows a simple linear model based on packet size $x$, and is $\alpha_{k} x+\beta_{k}$, where $\alpha_{k}$ and $\beta_{k}$ are parameters of module $k$ and are summarized in Table II. The link transmission time is proportional to the packet size, and the output bandwidth of the middlebox is $200 \mathrm{Mbps}$, the same as [10].

Fairness: We confirm experimentally that $\mathrm{GMR}^{3}$ provides near-perfect service isolation across flows, irrespective of their behaviours. The simulator generates 30 traffic flows that send 1300-byte UDP packets for 30 seconds. Flows 1 to 10 pass through the Basic module; flows 11 to 20 undergo statistical monitoring; while flows 21 to 30 require IPSec encryption. Among all these flows, flow 1, 11, and 21 are rogue traffic, each sending 30,000 pkts/s. All other flows behave normally, each sending 3,000 pkts/s. Flows are assigned random weights uniformly drawn from 1 to 1000 . Fig. 7a depicts the dominant services, in seconds, received by different flows under $\mathrm{GMR}^{3}$, normalized to their respective weights. We see that despite the presence of ill-behaving traffic, $\mathrm{GMR}^{3}$ allows flows through different modules to receive weight-proportional dominant services, enforcing service isolation. Similar results have also been observed using DRFQ and $\mathrm{MR}^{3}$, and are not shown in the figure.

Scheduling Delay: We next confirm experimentally that $\mathrm{GMR}^{3}$ significantly improves the packet scheduling delay, as compared to existing multi-resource scheduling alternatives. The simulator generates 150 UDP flows with flow weights uniformly drawn from 1 to 1000 . A flow randomly chooses one of the three middlebox modules to pass through. To congest the middlebox resources, the flow rate is set to $500 \mathrm{pkts} / \mathrm{s}$, with packet sizes uniformly drawn from $200 \mathrm{~B}$ to $1400 \mathrm{~B}$, which are the typical settings for Ethernet. For each processed packet, we record its scheduling delay, using DRFQ, $\mathrm{MR}^{3}$, and $\mathrm{GMR}^{3}$, respectively. The simulation spans 30 seconds.

Fig. 7b shows the CDF of the scheduling delay a packet experiences, from which we see the significance of $\mathrm{GMR}^{3}$ on delay improvement: Using $\mathrm{GMR}^{3}$, over $95 \%$ packets are scheduled within $20 \mathrm{~ms}$, which is roughly the minimum time a packet has to wait under DRFQ and $\mathrm{MR}^{3}$ ! A detailed statistics breakdown is given in Fig. 7c and 7d. Fig. 7c shows the mean scheduling delay a flow experiences with respective to its weight. We see that $\mathrm{GMR}^{3}$ consistently leads to a smaller mean delay than the other two schedulers for almost all flows, especially for those with large weights. This delay improvement is not limited to the average case. Fig. $7 d$ gives the maximum delay a flow experiences with respect to its weight. We see that both $\mathrm{GMR}^{3}$ and DRFQ offer a weight-proportional delay bound. While DRFQ achieves a smaller delay bound for flows with smaller weights, $\mathrm{GMR}^{3}$ is generally better for more important flows with medium to large weights. $\mathrm{MR}^{3}$, on the other hand, fails to provide service differentiations among flows. Intuitively, since flows 


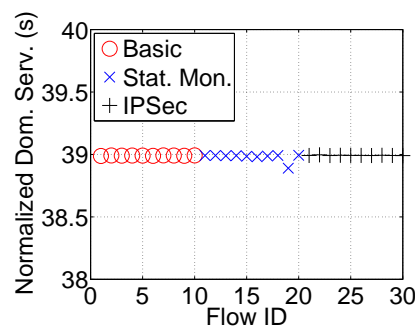

(a) Normalized dominant service.

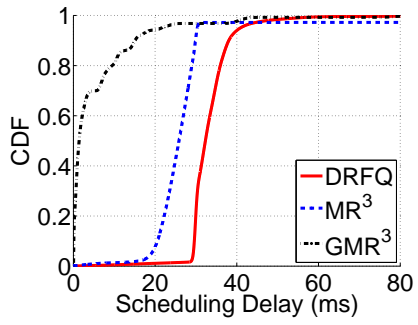

(b) CDF of the scheduling delay.

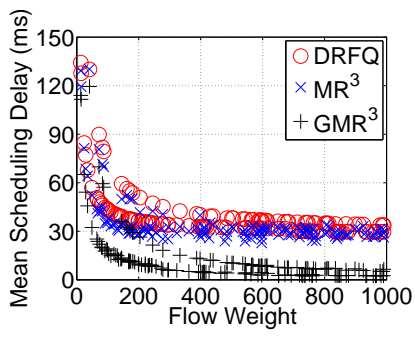

(c) Mean scheduling delay.

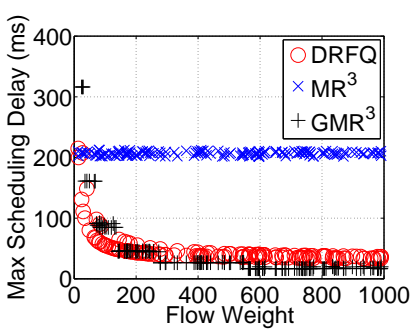

(d) Maximum scheduling delay.

Fig. 7. Simulation results of the fairness and delay performance of $\mathrm{GMR}^{3}$, as compared to DRFQ and $\mathrm{MR}^{3}$. Figure (a) dedicates to the fairness evaluation, while (b), (c), and (d) compare the scheduling delay of the three schedulers.

are served in rounds, in the worst case, a packet has to wait for the entire scheduling round until it is processed, incurring a worst-case delay that is as long as the span of an entire round. $\mathrm{GMR}^{3}$ avoids this problem by distributing the scheduling opportunities over time, in proportion to the flows' weights.

\section{CONCLUDING REMARKS}

In this paper, we design a new packet scheduler, called Group Multi-Resource Round Robin $\left(\mathrm{GMR}^{3}\right)$, that allows independent flows to have a fair share on multiple middlebox resources. GMR ${ }^{3}$ groups flows with similar weights to the same flow group, and makes scheduling decisions in a twolevel hierarchy. The inter-group scheduler determines a flow group, from which the intra-group scheduler picks a flow in a round-robin manner. Through this design, $\mathrm{GMR}^{3}$ eliminates the sorting bottlenecks suffered by existing multi-resource scheduling alternatives such as DRFQ, and is able to handle a large volume of traffic at high speeds. More importantly, we show, both analytically and experimentally, that $\mathrm{GMR}^{3}$ ensures constant scheduling delay bound that is inversely proportional to the flow's weight, hence offering predictable delay guarantees for individual flows. To our knowledge, $\mathrm{GMR}^{3}$ is the first multi-resource fair queueing algorithm that offers near-perfect fairness with a constant scheduling delay bound in $O(1)$ complexity.

\section{REFERENCES}

[1] A. Demers, S. Keshav, and S. Shenker, "Analysis and simulation of a fair queueing algorithm," in Proc. ACM SIGCOMM, 1989.

[2] A. Parekh and R. Gallager, "A generalized processor sharing approach to flow control in integrated services networks: The single-node case," IEEE/ACM Trans. Netw., vol. 1, no. 3, pp. 344-357, 1993.

[3] H. Zhang, "Service disciplines for guaranteed performance service in packet-switching networks," Proc. IEEE, vol. 83, no. 10, pp. 1374-1396, 1995.

[4] M. Shreedhar and G. Varghese, "Efficient fair queuing using deficit round-robin," IEEE/ACM Trans. Netw., vol. 4, no. 3, pp. 375-385, 1996.

[5] P. Goyal, H. Vin, and H. Cheng, "Start-time fair queueing: A scheduling algorithm for integrated services packet switching networks," IEEE/ACM Trans. Netw., vol. 5, no. 5, pp. 690-704, 1997.

[6] A. Greenhalgh, F. Huici, M. Hoerdt, P. Papadimitriou, M. Handley, and L. Mathy, "Flow processing and the rise of commodity network hardware," ACM SIGCOMM Comput. Commun. Rev., vol. 39, no. 2, pp. 20-26, 2009.

[7] J. Sherry, S. Hasan, C. Scott, A. Krishnamurthy, S. Ratnasamy, and V. Sekar, "Making middleboxes someone else's problem: Network processing as a cloud service," in Proc. ACM SIGCOMM, 2012.

[8] J. Anderson, R. Braud, R. Kapoor, G. Porter, and A. Vahdat, "xOMB: Extensible open middleboxes with commodity servers," in Proc. ACM/IEEE ANCS, 2012.
[9] V. Sekar, N. Egi, S. Ratnasamy, M. Reiter, and G. Shi, "Design and implementation of a consolidated middlebox architecture," in Proc. USENIX NSDI, 2012

[10] A. Ghodsi, V. Sekar, M. Zaharia, and I. Stoica, "Multi-resource fair queueing for packet processing," in Proc. ACM SIGCOMM, 2012.

[11] S. Golestani, "A self-clocked fair queueing scheme for broadband applications," in Proc. IEEE INFOCOM, 1994.

[12] J. Bennett and $\mathrm{H}$. Zhang, "WF' ${ }^{2} \mathrm{Q}$ : Worst-case fair weighted fair queueing," in Proc. IEEE INFOCOM, 1996.

[13] N. Egi, A. Greenhalgh, M. Handley, M. Hoerdt, F. Huici, and L. Mathy, "Towards high performance virtual routers on commodity hardware," in Proc. ACM CoNEXT, 2008.

[14] H. Dreger, A. Feldmann, V. Paxson, and R. Sommer, "Predicting the resource consumption of network intrusion detection systems," in Recent Advances in Intrusion Detection (RAID), vol. 5230. Springer, 2008, pp. $135-154$.

[15] M. Honda, Y. Nishida, C. Raiciu, A. Greenhalgh, M. Handley, and H. Tokuda, "Is it still possible to extend TCP?" in Proc. ACM IMC, 2011.

[16] Z. Wang, Z. Qian, Q. Xu, Z. Mao, and M. Zhang, "An untold story of middleboxes in cellular networks," in Proc. SIGCOMM, 2011.

[17] A. Ghodsi, M. Zaharia, B. Hindman, A. Konwinski, S. Shenker, and I. Stoica, "Dominant resource fairness: Fair allocation of multiple resource types," in Proc. USENIX NSDI, 2011.

[18] W. Wang, B. Li, and B. Liang, "Multi-resource round robin: A low complexity packet scheduler with dominant resource fairness," in Proc. IEEE ICNP, 2013.

[19] C. Joe-Wong, S. Sen, T. Lan, and M. Chiang, "Multi-resource allocation: Fairness-efficiency tradeoffs in a unifying framework," in Proc. IEEE INFOCOM, 2012.

[20] D. Parkes, A. Procaccia, and N. Shah, "Beyond dominant resource fairness: Extensions, limitations, and indivisibilities," in Proc. ACM EC, 2012.

[21] W. Wang, B. Liang, and B. Li, "Multi-resource generalized processor sharing for packet processing," in Proc. ACM/IEEE IWQoS, 2013.

[22] S. Ramabhadran and J. Pasquale, "Stratified round robin: A low complexity packet scheduler with bandwidth fairness and bounded delay," in Proc. ACM SIGCOMM, 2003.

[23] S. Kanhere, H. Sethu, and A. Parekh, "Fair and efficient packet scheduling using elastic round robin," IEEE Trans. Parallel Distrib. Syst., vol. 13, no. 3, pp. 324-336, 2002.

[24] B. Caprita, J. Nieh, and W. C. Chan, "Group round robin: Improving the fairness and complexity of packet scheduling," in Proc. ACM ANCS, 2005.

[25] X. Yuan and Z. Duan, "Fair round-robin: A low-complexity packet scheduler with proportional and worst-case fairness," IEEE Trans. Comput., 2009.

[26] W. Wang, B. Liang, and B. Li, "Multi-resource fair queueing for packet processing with low complexity and bounded delay," University of Toronto, Tech. Rep., 2013. [Online]. Available: http://iqua.ece.toronto.edu/ weiwang/papers/gmr3.pdf

[27] S. Floyd and V. Jacobson, "Link-sharing and resource management models for packet networks," IEEE/ACM Trans. Netw., vol. 3, no. 4 , pp. 365-386, 1995.

[28] S. Y. Cheung and C. S. Pencea, "BSFQ: Bin sort fair queueing," in Proc. IEEE INFOCOM, 2002.

[29] C. Guo, "SRR: An $O(1)$ time complexity packet scheduler for flows in multi-service packet networks," in Proc. ACM SIGCOMM, 2001. 